OPEN ACCESS

Edited by:

Hui Wang,

Hunan Cancer Hospital, China

Reviewed by:

Shahram Ghanaati,

Goethe University Frankfurt, Germany

Vito Carlo Alberto Caponio,

University of Foggia, Italy

*Correspondence:

Yu Zhao

yutzhao@VIP.163.com

Jianjun Ren

juneent2016@163.com

${ }^{t}$ These authors have contributed equally to this work

Specialty section:

This article was submitted to

Head and Neck Cancer,

a section of the journal

Frontiers in Oncology

Received: 18 May 2021 Accepted: 01 September 2021 Published: 27 September 2021

Citation:

Qiu K, Song Y, Rao Y, Liu Q, Cheng D,

Pang W, Ren J and Zhao Y (2021)

Diagnostic and Prognostic Value of

MicroRNAs in Metastasis and

Recurrence of Head and Neck

Squamous Cell Carcinoma: A

Systematic Review and Meta-Analysis.

Front. Oncol. 11:711171.

doi: 10.3389/fonc.2021.711171

\section{Diagnostic and Prognostic Value of MicroRNAs in Metastasis and Recurrence of Head and Neck Squamous Cell Carcinoma: A Systematic Review and Meta-Analysis}

Ke Qiu ${ }^{1+}$, Yao Song ${ }^{1+}$, Yufang Rao ${ }^{1+}$, Qiurui Liu ${ }^{1}$, Danni Cheng ${ }^{1}$, Wendu Pang ${ }^{1}$, Jianjun Ren ${ }^{1,2,3^{*}}$ and Yu Zhao ${ }^{1 *}$

\begin{abstract}
${ }^{1}$ Department of Oto-Rhino-Laryngology, West China Hospital, Sichuan University, Chengdu, China, ${ }^{2}$ West China Biomedical Big Data Center, West China Hospital/West China School of Medicine, Sichuan University, Chengdu, China, ${ }^{3}$ Medical Big Data Center, Sichuan University, Chengdu, China
\end{abstract}

MicroRNAs have been proven to make remarkable differences in the clinical behaviors of head and neck squamous cell carcinoma (HNSCC). This study aims to systematically analyze whether differential expression levels of microRNAs are related to recurrence or metastasis in patients with HNSCC. A comprehensive search of the PubMed, EMBASE, and CENTRAL was conducted up to July $24^{\text {th }}, 2021$. Data were collected and combined from studies reporting recurrence-free survival (RFS) of HNSCC patients with high microRNA expression compared to those with low expression. Besides, studies providing necessary data for evaluating the diagnostic value of microRNAs for detecting recurrence and metastasis based on their expression levels were also included and combined. The pooled hazard ratio (HR) value for the outcomes of RFS in 1,093 HNSCC samples from 10 studies was 2.51 (95\% Cl: 2.13-2.96). A sensitivity of 0.79 (95\% Cl: $0.72-0.85)$ and specificity of 0.77 (95\% Cl: 0.68-0.83) were observed in three studies, of which 93 patients with recurrence and 82 nonrecurrence controls were included, and the area under the curve (AUC) was 0.85 (95\% Cl: 0.81-0.88). Additionally, high diagnostic accuracy of microRNAs in detecting lymph node metastasis (LNM) was also reported. In conclusion, two panels of microRNAs showed the potential to predict recurrence or diagnose recurrence in HNSCC patients, respectively, which could facilitate prognosis prediction and diagnosis of clinical behaviors in HNSCC patients.

Systematic Review Registration: PROSPERO (https://www.crd.york.ac.uk/prospero), identifier CRD42020161117.

Keywords: microRNA, head and neck squamous cell carcinoma (HNSCC), recurrence-free survival (RFS), metastasis, diagnostic accuracy, meta-analysis 


\section{INTRODUCTION}

Head and neck squamous cell carcinomas (HNSCCs), visualized as the most common form of human solid tumors in the head and neck region, account for a large proportion of cancer mortality worldwide $(1,2)$. A multiple process that accumulates genetic mutations sequentially is believed to play a critical role in the formation of HNSCCs. And these highly heterogeneous tumors are derived from stratified epithelial cells of various anatomical subsites, mainly including the oral cavity, tongue, nasal cavity, larynx, and pharynx $(3,4)$. However, recent studies had illustrated similarities in genomic, genetic, and epigenetic alterations between HNSCCs from different subsites, which suggested the existence of certain common mechanisms underlying the initiation and progression of $\operatorname{HNSCCs}(3,5,6)$. It is estimated that approximately $1 / 3$ of HNSCC patients develop recurrence or metastasis after receiving standard therapies, and the majority of them ended with poor prognosis $(7,8)$. Currently, tumor-node-metastasis (TNM) staging based on imaging modalities and biopsy represents the leading way to predict HNSCCs' biological behaviors, especially for metastasis and recurrence; however, accuracy varied among HNSCCs with different origins $(9,10)$. Therefore, reliable and detectable biomarkers may contribute to the diagnosis and prediction of metastasis and recurrence of SCCs.

MicroRNAs, a special class of noncoding RNAs (19-23 nucleotides in length), are capable of binding to their target mRNAs and regulating gene expression at the posttranscriptional level (11). In the past decade, microRNAs had been proven to make remarkable differences in HNSCC carcinogenesis and cancer progression, making them potential biomarkers to predict biological behaviors of HNSCCs (12-18). This meta-analysis aimed at evaluating the diagnostic and prognostic values of microRNAs in the recurrence and metastasis of human SCCs.

\section{METHODS AND MATERIALS}

\section{Protocol and Eligibility Criteria}

This study was conducted in accordance with the PRISMA (Preferred Reporting Items for Systematic Reviews and MetaAnalysis) guidelines (19) and was already registered on prospero (registration number: CRD42020161117).

The inclusion criteria were as follows: 1) studies written in English; 2) the diagnosis of HNSCC was confirmed by histopathology; 3) studies demonstrating the expression levels of microRNA by quantitative polymerase chain reaction (qPCR), in situ hybridization (ISH), fluorescent in situ hybridization (FISH), or RNA sequencing; 4) studies reporting HR with 95\% confidence interval (CI) or Kaplan-Meier curves related to the correlation of microRNA expression with RFS; and 5) studies providing necessary data for evaluating the diagnostic value of microRNAs in predicting recurrence and metastasis.

The exclusion criteria were as follows: 1) studies related to nonhuman samples; 2) studies providing combined outcomes of more than one microRNA or insufficient data; 3) duplicate studies; 4) studies in the form of reviews, letters, editorials, meeting abstracts, or case reports; and 5) studies reporting SCC not originated from the head and neck region.

More specifically, for the meta-analysis evaluating the predictive values of microRNAs, we aimed to address the question of whether HNSCC patients with a higher expression level of microRNAs had different risk of cancer recurrence compared to HNSCC patients with a lower expression level of microRNAs. And for the meta-analysis evaluating the diagnostic values of microRNAs, we aimed to address the question of whether the expression level of microRNAs in human biospecimen could be used as indicators for distinguishing the HNSCC patients with recurrence/metastasis or not.

\section{Information Sources and Search Strategies}

A comprehensive search of the PubMed, EMBASE, and CENTRAL was conducted from the beginning of each database to July $24^{\text {th }}, 2021$. Meanwhile, additional records from other sources, e.g., the references of included studies and original studies mentioned in reviews, were also screened.

A Boolean combination of Mesh terms and free text words were used as search strategies, mainly including "Carcinoma, Squamous Cell"[Mesh], "MicroRNA", "MiRNAs", "MicroRNAs”[Mesh], "Metastasis", "Metastases", "Recurrences", "Relapse", and "Recurrence"[Mesh]. Detailed search strategies were presented in Supplementary Material.

\section{Study Selection, Data Collection Process, and Data Items}

The initial screening of titles and abstracts were conducted independently by two authors, and full texts would be reviewed if the titles and abstracts were ambiguous. Any discrepancy was solved by consensus, and a third author would participate if necessary. Finally, studies fulfilling all inclusion criteria were included in the systematic review.

Data extraction for studies investigating the RFS and diagnostic value was also independently performed and crosschecked by two authors. The agreement between authors KQ and YS was determined by Cohen's kappa score. And the extracted items were as follows: year of publication, first author, country, microRNAs studied, type of microRNA dysregulation, sample sizes, duration of follow-up, tumor sites, metastasis sites, detection assay, sample types, and cutoffs for the expression of microRNAs.

\section{Risk of Bias Assessment, Summary Measures, and Statistical Analyses}

The risk of bias within each included prognostic study was evaluated by the Newcastle-Ottawa Scale (NOS) (http://www. ohri.ca/programs/clinical_epidemiology/oxford.asp), and the risk of bias within each included diagnostic study was evaluated by the tool provided by Quality Assessment of Diagnostic Accuracy Studies-2 (QUADAS-2) (20), while the risk of bias across studies was evaluated by the Cochran Q test 
and Higgins index $\left(I^{2}\right)$. Heterogeneity was considered significant if $P<0.05$ in $\mathrm{Q}$ test, and subgroup analyses were applied to find the potential sources of heterogeneity. Besides, overall effects were analyzed by a fixed-effect model if $I^{2}<50 \%$; otherwise, a random-effects model would be applied.

For studies investigating RFS, $\ln [\mathrm{HR}]$ and standard error (SE) were synthesized (21). The publication bias was tested by Begg's test. For studies investigating diagnostic values, we calculated the pooled sensitivity and specificity. The summary receiver operator characteristic (SROC) curve and the area under the SROC curve (AUC) were constructed and calculated to explore the diagnostic accuracy of microRNAs in metastasis/recurrence. Additionally, the publication bias was tested by Deek's funnel plot asymmetry test.

The STATA 12.0 (Stata Corp, College Station, TX, USA) and Review Manager (Version5.4, The Cochrane Collaboration, 2020) software was used for all meta-analysis, and $P<0.05$ was considered significant.

\section{RESULTS}

\section{Study Selection}

A total of 3,349 records were retrieved through the initial comprehensive search. A total of 1,860 articles remained after excluding duplicates and were screened according to titles and abstracts. Subsequently, 398 studies remained and underwent full-text screening. A total of 14 studies that fully met the inclusion criteria were included for further analysis, among which 10 studies (22-31) were included for meta-analysis of HR for RFS, 3 studies (32-34) were included for meta-analysis of diagnostic accuracy for recurrence, and another 1 study (14) was included for systematic review of diagnostic accuracy for metastasis. Detailed selection process and reasons of exclusion were presented in Figure 1.

\section{Study Characteristics}

For the 10 included studies investigating RFS, a cumulative number of 1,093 SCC samples were analyzed. Tumor subsites were confined to head and neck regions, including the oral cavity, larynx, hypopharynx, and oropharynx. The duration of follow-up ranged from 28 months to more than 120 months, and all outcomes were evaluated by multivariate Cox regression models. Detailed characteristics of each study are presented in Table 1.

For the three included studies investigating diagnostic accuracy for recurrence, a total of 93 recurrence patients and 82 nonrecurrence controls were analyzed. Tumor subsites were confined to head and neck regions, including larynx and oral cavity. And the recurrence sites were not reported in most of the studies. Detailed characteristics of each study are presented in Table 2.

Similarly, for the included study investigating diagnostic accuracy for metastasis, a total of 25 metastasis patients and 23 nonmetastasis controls were analyzed. Tumor subsites were confined to head and neck regions. And the metastasis sites were reported to be the lymph node. Detailed characteristics of each study are presented in Table 2 .

\section{Synthesis of RFS and Subgroup Analysis}

The pooled HR value for the outcomes of RFS in all HNSCC patients was 2.51 (95\%CI: 2.13-2.96) (Figure 2). Poorer RFS correlated with upregulation of 14 microRNAs (miR-205-5p, miR-429, miR-21-3p, miR-331-3p, miR-200a-3p, miR-19a-3p, miR-21-5p, miR-151a-3p, miR-17-3p, miR-18b-5p, miR-324-5p, miR-96-5p, miR-141-3p, and miR-130a) and with downregulation of 7 microRNAs (miR-29c, miR-200b, miR-375, miR-422a, miR-15b-5p, miR-204, and miR-200c).

We further conducted a subgroup analysis based on anatomical subsites, and the results showed that pooled HR values for the outcomes of RFS were 2.02 (95\%CI: 1.10-3.71) in oropharyngeal squamous cell carcinoma (OPSCC) patients and 2.12 (95\%CI: 1.642.73 ) in oral squamous cell carcinoma (OSCC) patients (Figure 3). And in OPSCC patients, poorer RFS correlated with downregulation of miR-422a and miR-375. While in OSCC patients, poorer RFS correlated with upregulation of five microRNAs (miR-21-3p, miR-130b-3p, miR-96-5p, miR-141-3p, and miR-130a) and with downregulation of three microRNAs (miR-375, miR-204, and miR-200c) (Figure 3A). Meanwhile, we also conducted a subgroup analysis for individual microRNAs with more than two outcomes. And the expression levels of miR-21-3p, miR-96-5p, and miR-375 showed strong association with RFS, especially miR-21-3p, with a pooled HR value of 3.59 (95\%CI: 1.91-6.76) (Figure 3B).

Taken together, our results showed that a panel of 21 microRNAs (miR-205-5p, miR-429, miR-21-3p, miR-331-3p, miR-200a-3p, miR-19a-3p, miR-21-5p, miR-151a-3p, miR-173p, miR-18b-5p, miR-324-5p, miR-96-5p, miR-141-3p, miR130a, miR-29c, miR-200b, miR-375, miR-422a, miR-15b-5p, miR-204, and miR-200c) might have the potential to predict the prognosis of patients with HNSCCs, of which 2 microRNAs were associated with the prognosis of OPSCC patients, and 8 microRNAs were associated with the prognosis of OSCC patients.

\section{Study Quality and Risk of Bias in Prognostic Studies}

NOS scores of each prognostic study are listed in Table S1, of which eight studies (80\%) were of "good" quality and deemed to have low risk of bias, while the other two studies (20\%) were of "poor" quality and deemed to have high risk of bias mainly due to the lack of adjustment for important confounding variables. A Cohen's kappa score of 0.76 revealed great agreement beyond chance between the two authors. Besides, no statistically significant heterogeneity $(P>0.1)$ and publication bias (Begg's test: $P=0.333$, Figure $\mathbf{S 1}$ ) was observed in the pooled analysis of RFS.

\section{Pooled Diagnostic Accuracy of microRNAs for HNSCC Recurrence}

The evaluation for the sensitivity and specificity of a panel of four microRNAs in diagnosing the recurrence of SCCs is illustrated in Figure 4A (miR-34c-5p, miR-186-5p, miR-3651, 


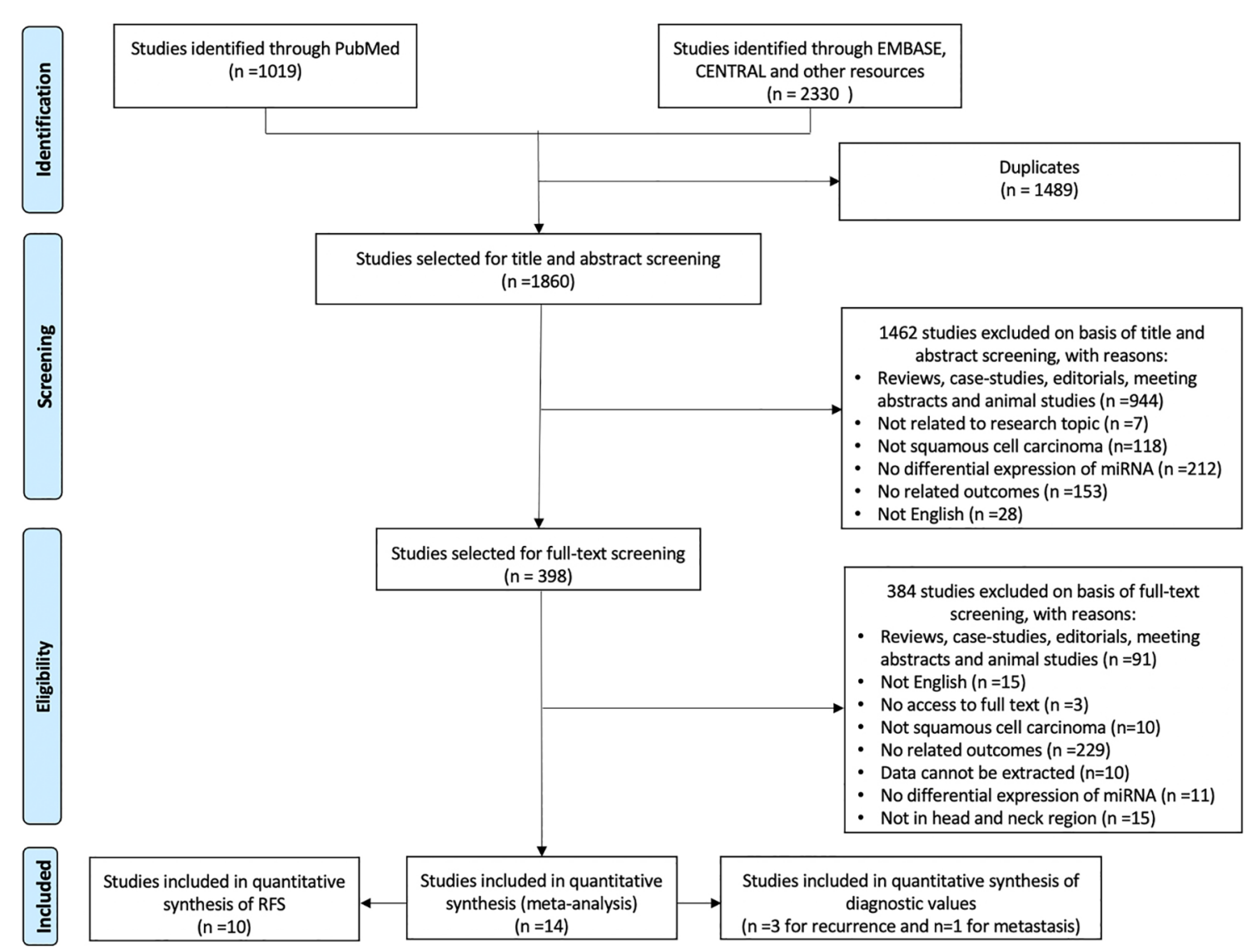

FIGURE 1 | Flow diagram summarizing the selection process of the systematic review.

and miR-494-5p). A sensitivity of 0.79 (95\% CI: 0.72-0.85) and a specificity of 0.77 ( $95 \%$ CI: $0.68-0.83$ ) were observed in patients with recurrence and nonrecurrence controls. The AUC was 0.85 (95\% CI: 0.81-0.88), and the corresponding SROC curve is presented in Figure 4B.

\section{Study Quality and Risk of Bias in Diagnostic Studies}

As is shown in Figure S2, nearly all included diagnostic studies showed low risk of bias in patient selection, reference standard, and flow and timing; however, all of these three studies showed high risk of bias in index test mainly due to their retrospective nature, in which the index test results cannot be interpreted without knowledge of the results of the reference standard. Besides, the diagnostic thresholds in these studies were not confined and also not prespecified, which might cause some concerns on applicability. Meanwhile, no significant heterogeneity $(P>0.1)$ and publication bias $(P=0.53$, Figure S3) was observed.

\section{Overview of Diagnostic Accuracy of microRNAs for LNM in HNSCC}

Only one study reporting the diagnostic accuracy of four independent microRNAs for LNM in HNSCC met our eligibility criteria. However, given the high risk of bias for pooled analysis of four tests derived from the same study, meta-analysis was not conducted. de Carvalho et al. reported the diagnostic accuracy of miRNA-200a (sensitivity 0.76; specificity:0.88; AUC: 0.92), miRNA-200c (sensitivity: 0.88; specificity: 1.00; AUC: 0.94), miRNA-203 (sensitivity: 1.00; specificity: 1.00; AUC: 1.00), and miRNA-205 (sensitivity: 1.00; specificity: 1.00; AUC: 1.00) in distinguishing 25 HNSCC patients with LNM from 23 HNSCC patients without LNM (14). All of these four microRNAs showed high diagnostic accuracy for detecting LNM in HNSCC patients; however, their performance was evaluated in the same cohort of which the sample size is not large enough. Thus, whether microRNAs have the potential for detecting LNM in HNSCC still needs to be validated by more studies with larger sample sizes.

\section{DISCUSSION}

In this review, we systematically analyzed 1,093 HNSCC samples from 10 studies (22-31) and identified a panel of 21 microRNAs related to poor RFS in HNSCC patients for the first time. Besides, we investigated the diagnostic accuracy of microRNAs for 
TABLE 1 | Detailed characteristics and NOS scores of included studies investigating RFS.

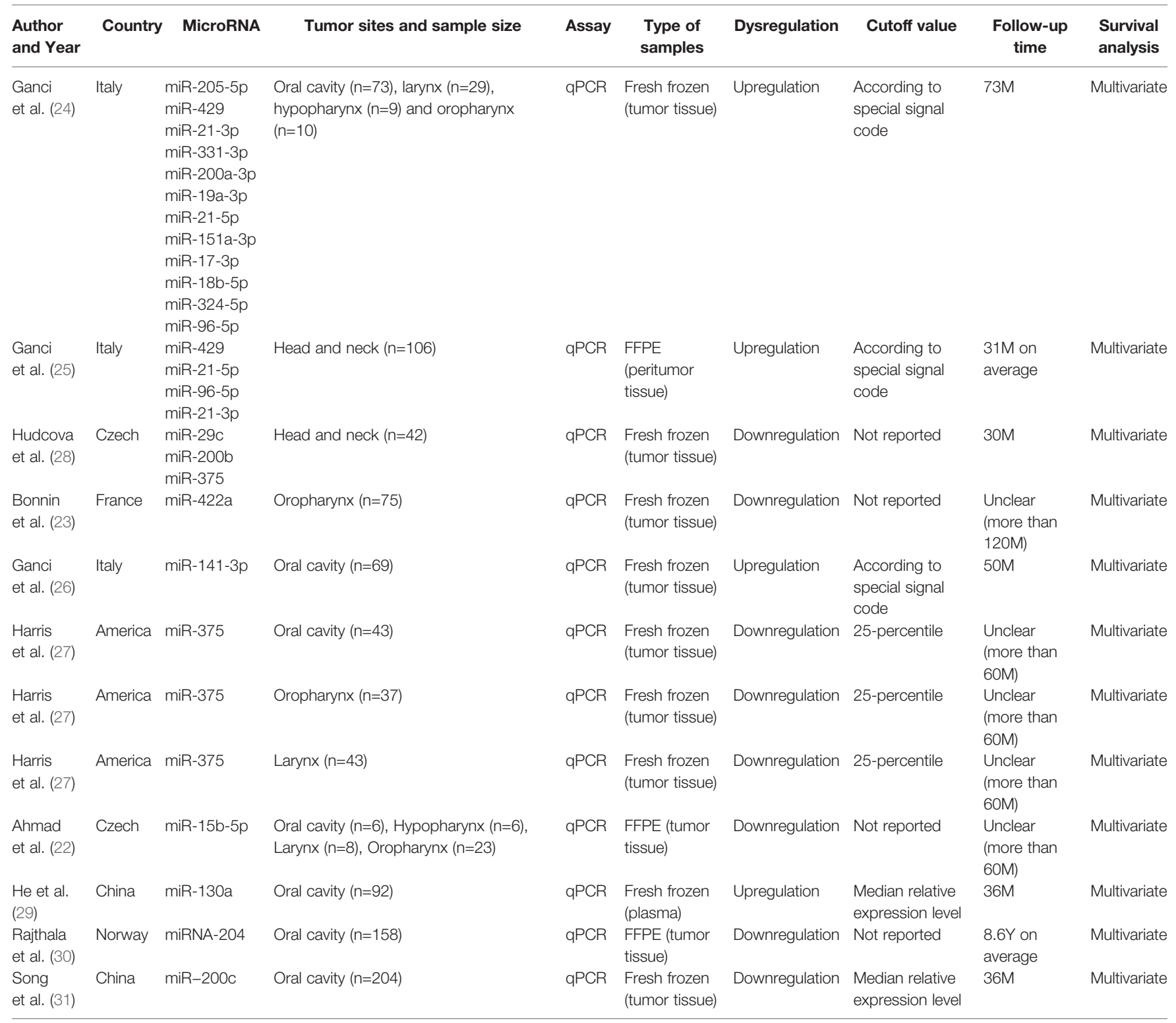

TABLE 2 | Detailed characteristics of included studies investigating diagnostic values of microRNAs for recurrence and LNM.

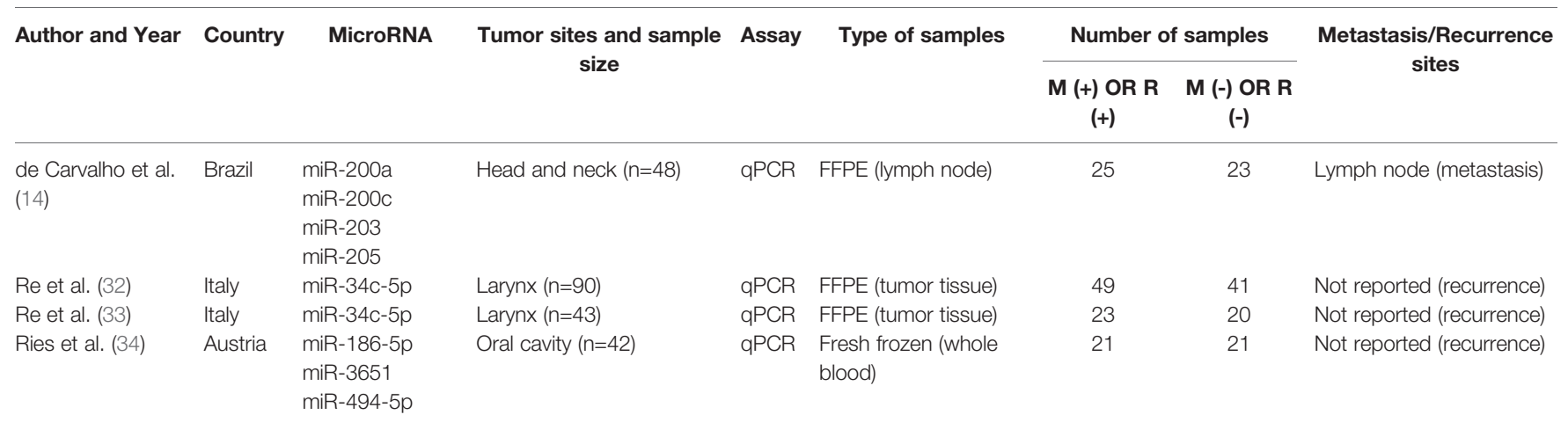




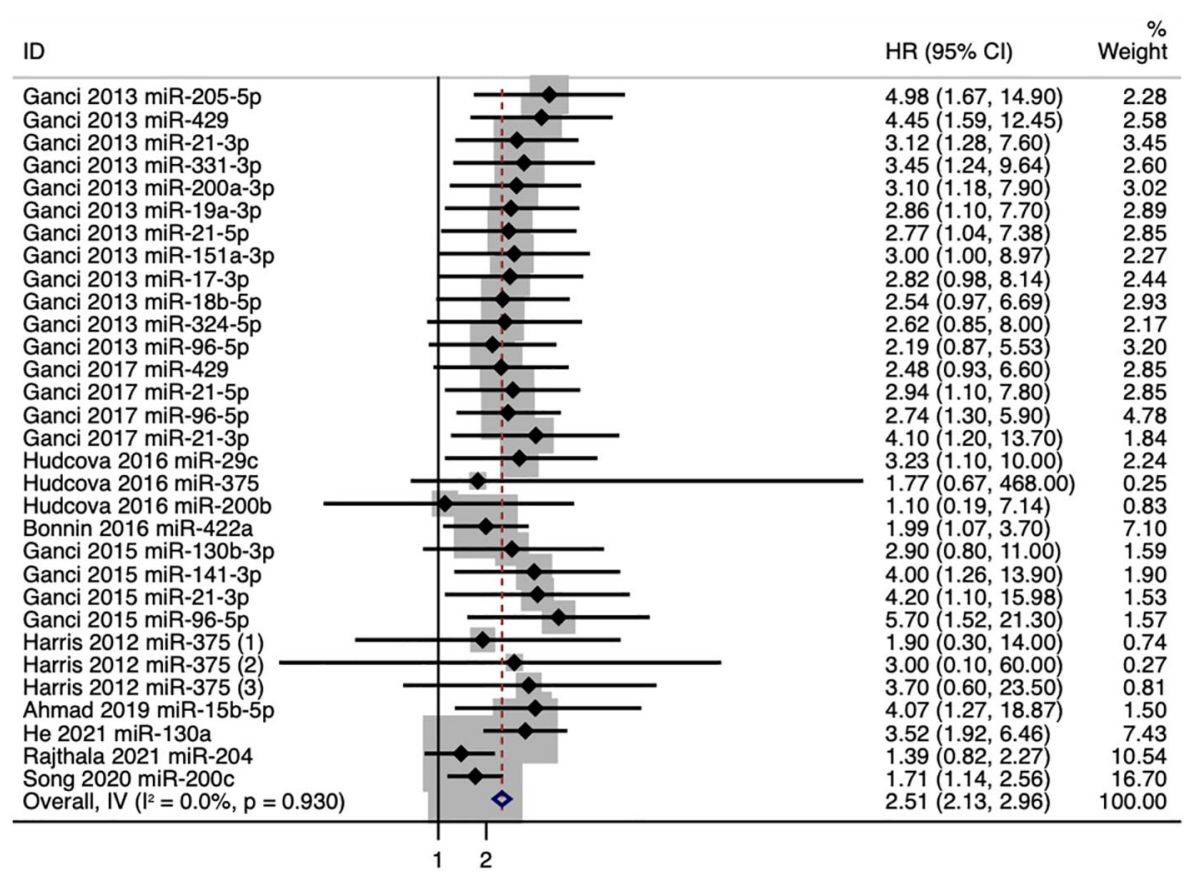

FIGURE 2 | Forest plot for the association between microRNA expression and recurrence-free survival (RFS).

A

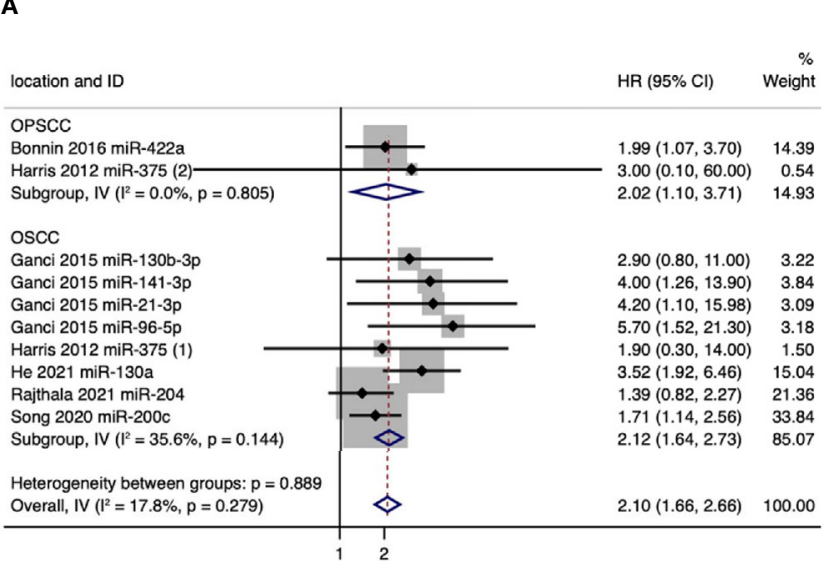

B

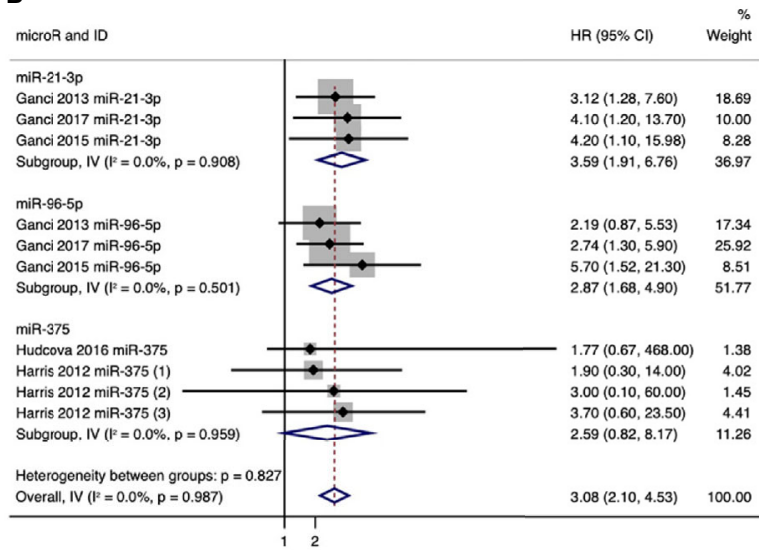

FIGURE 3 | (A) Forest plot for subgroup analysis of the association between microRNA expression and recurrence-free survival (RFS) based on anatomical subsites. (B) Forest plot for subgroup analysis of the association between microRNA expression and recurrence-free survival (RFS) based on microRNAs (only individual microRNAs with more than two outcomes were presented).

recurrence (by analyzing 93 recurrence patients and 82 nonrecurrence controls from 3 studies) (32-34) and LNM (by presenting an overview of 25 metastasis patients and 23 nonmetastasis controls from another study) (14). We observed relatively high diagnostic accuracy of microRNAs in diagnosing recurrence and LNM for HNSCCs, which have the potential to assist imaging modalities and histopathology biopsy in the diagnosis and prognosis for HNSCC patients.
In the current study, we specifically took recurrence and metastasis as the outcomes. Our results showed that a panel of 21 microRNAs might be suitable biomarkers for predicting the recurrence of HNSCCs (pooled HR:2.51, 95\%CI: 2.13-2.96, $I^{2}=0$ ), among which poor RFS correlated with upregulation of 14 microRNAs (miR-205-5p, miR-429, miR-21-3p, miR-331-3p, miR-200a-3p, miR-19a-3p, miR-21-5p, miR-151a-3p, miR-173p, miR-18b-5p, miR-324-5p, miR-96-5p, miR-141-3p, and 


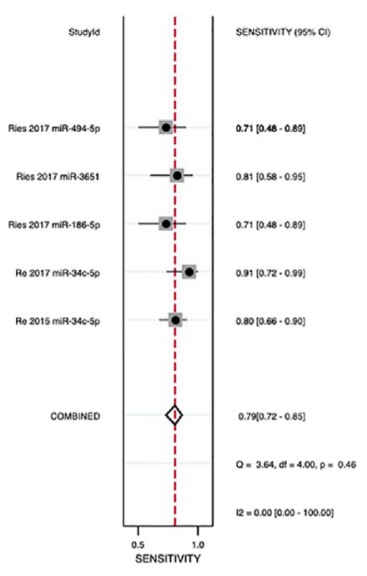

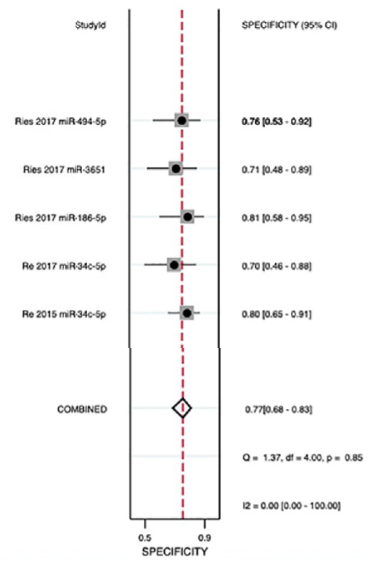

B

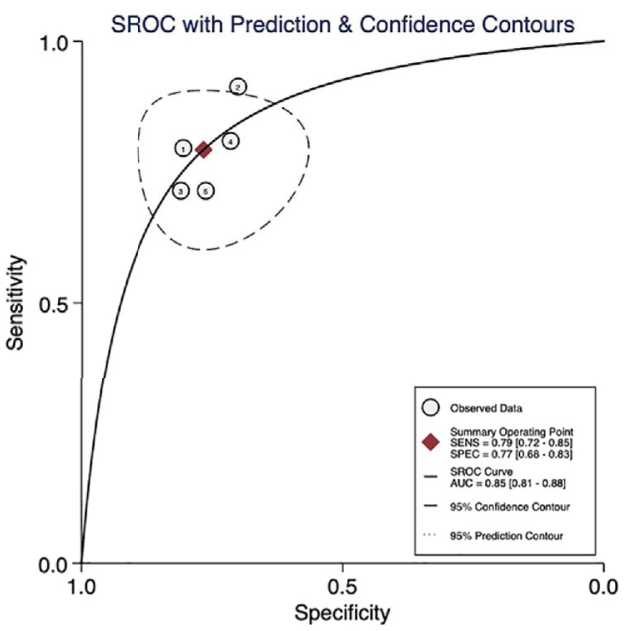

FIGURE 4 | (A) Sensitivity and specificity of microRNAs in diagnosing recurrence. (B) The summary receiver operating characteristic (SROC) curves of the diagnostic performance of microRNAs for recurrence.

miR-130a) or with downregulation of 7 microRNAs (miR-29c, miR-200b, miR-375, miR-422a, miR-15b-5p, miR-204, and miR200c). Besides, subgroup analyses revealed similar trends in OSCC and OPSCC patients as well as identified miR-21-3p, miR-96-5p, and miR-375, which showed strong association with RFS. Our quality assessments showed that $80 \%$ of the included studies investigating RFS were of good quality, and the meta-analysis was of low risk of bias, which reinforced the reliability of our results. Meanwhile, the diagnostic accuracy of microRNAs in HNSCC recurrence was decent (sensitivity: 0.79 , 95\% CI: $0.72-$ 0.85; specificity: 0.77, 95\%CI: 0.68-0.83; AUC: 0.85 , 95\% CI: $0.81-$ 0.88 ), together with a low rate of heterogeneity and low risk of publication bias. Additionally, the diagnostic accuracy of microRNAs in LNM of HNSCC reported by de Carvalho et al. was relatively high but needs further validation.

We further summarized these analyzed microRNAs and explored their well-known biological functions related to tumor malignant behaviors in Table S2. As expected, the downregulation of microRNAs, which had been previously reported to be related to tumor suppressing functions, including inhibiting tumor cell proliferation, migration, and invasion (18, 35-61), was associated with poorer RFS in our study. Similarly, the upregulation of microRNAs, which had been previously reported to function as enhancers of tumor malignant behaviors (62-83), were associated with poorer RFS. Besides, the modulation of epithelial to mesenchymal transition (EMT), an essential early step of tumor metastasis, was identified as the most commonly reported mechanism, through which those microRNAs regulate malignant behaviors of other types of tumor. Meanwhile, surprisingly, we found five members of the microRNA-200 family (miR-200a, miR-200b, miR-200c, miR-429, and miR-141), which are known for their regulatory function on EMT, and their combination might act as a strong prognosis predictor (Figure S3) (39, 84-88). Thus, we hypothesized that the modulation of EMT and subsequent cell migration and invasion might also act as the key pathophysiological mechanisms of the interplay between microRNAs and HNSCC, which still needs to be further confirmed by mechanistical studies.

There were some limitations in this study. First, given the fact that most of the included studies performed their analyses in HNSCC samples with mixed subsites, which cannot be strictly separated, therefore subgroup analysis could only be available in OSCC and OPSCC patients. And consequently, whether our conclusions can be generalized to SCC derived from other subsites remained to be verified by further studies with larger sample sizes and a wider spectrum of HNSCC subsites. Second, it is also a pity that most of these included studies have not performed subgroup analyses based on HPV status; thus, we were not able to properly investigate the influence of the HPV status. However, most of these included studies have adjusted the HPV status in their multivariate analysis, which might reduce its influence to some extent. Third, the outcomes of four independent microRNAs for LNM in HNSCC were retrieved from the same study. Thus, pooled analysis was not conducted due to the potential high risk of bias. And the reported high diagnostic accuracy still needs to be further validated. Additionally, our results should be explained cautiously since our panels have significance when these microRNAs are taken as a whole, not individually.

\section{DATA AVAILABILITY STATEMENT}

The datasets generated for this study are available on request to the corresponding authors. 


\section{AUTHOR CONTRIBUTIONS}

JR and YZ contributed to the study conception and design. Material preparation was performed by $\mathrm{QL}$ and WP, data collection was performed by YR, and analysis was performed by KQ, YS, YR, and DC. The first draft of the manuscript was written by KQ and YS, and all authors commented on previous versions of the manuscript. All authors contributed to the article and approved the submitted version.

\section{FUNDING}

This work was supported by West China Hospital, Sichuan University (YZ, grant \# 2019HXFH003, grant \# ZYJC21027); Chengdu Science and Technology Bureau (JR, grant \# 20GJHZ0193); Sichuan University (YZ, grant \# 20ZDYF3010,

\section{REFERENCES}

1. Torre LA, Bray F, Siegel RL, Ferlay J, Lortet-Tieulent J, Jemal A. Global Cancer Statistic. CA Cancer J Clin (2015) 65(2):87-108. doi: 10.3322/caac.21262

2. Torre LA, Siegel RL, Ward EM, Jemal A. Global Cancer Incidence and Mortality Rates and Trends-An Update. Cancer Epidemiol Biomarkers Prev Publ Am Assoc Cancer Research cosponsored by Am Soc Prev Oncol (2016) 25 (1):16-27. doi: 10.1158/1055-9965.EPI-15-0578

3. Sanchez-Danes A, Blanpain C. Deciphering the Cells of Origin of Squamous Cell Carcinomas. Nat Rev Cancer (2018) 18(9):549-61. doi: 10.1038/s41568018-0024-5

4. Alsahafi E, Begg K, Amelio I, Raulf N, Lucarelli P, Sauter T, et al. Clinical Update on Head and Neck Cancer: Molecular Biology and Ongoing Challenges. Cell Death Dis (2019) 10(8):540. doi: 10.1038/s41419-019-1769-9

5. Dotto GP, Rustgi AK. Squamous Cell Cancers: A Unified Perspective on Biology and Genetics. Cancer Cell (2016) 29(5):622-37. doi: 10.1016/ j.ccell.2016.04.004

6. Mathelier A, Shi W, Wasserman WW. Identification of Altered CisRegulatory Elements in Human Disease. Trends Genet (2015) 31(2):67-76. doi: 10.1016/j.tig.2014.12.003

7. Argiris A, Karamouzis MV, Raben D, Ferris RL. Head and Neck Cancer. Lancet (2008) 371(9625):1695-709. doi: 10.1016/S0140-6736(08)60728-X

8. Svider PF, Blasco MA, Raza SN, Shkoukani M, Sukari A, Yoo GH, et al. Head and Neck Cancer. Otolaryngol Head Neck Surg (2017) 156(1):10-3. doi: $10.1177 / 0194599816674672$

9. Huang SH, O'Sullivan B. Overview of the 8th Edition TNM Classification for Head and Neck Cancer. Curr Treat Options Oncol (2017) 18(7):40. doi: 10.1007/s11864-017-0484-y

10. Denaro N, Russi EG, Merlano MC. Pros and Cons of the New Edition of TNM Classification of Head and Neck Squamous Cell Carcinoma. Oncology (2018) 95(4):202-10. doi: 10.1159/000490415

11. Jonas S, Izaurralde E. Towards a Molecular Understanding of microRNAMediated Gene Silencing. Nat Rev Genet (2015) 16(7):421-33. doi: 10.1038/ nrg3965

12. Benaich N, Woodhouse S, Goldie SJ, Mishra A, Quist SR, Watt FM. Rewiring of an Epithelial Differentiation Factor, miR-203, to Inhibit Human Squamous Cell Carcinoma Metastasis. Cell Rep (2014) 9(1):104-17. doi: 10.1016/ j.celrep.2014.08.062

13. Chang WM, Lin YF, Su CY, Peng HY, Chang YC, Lai TC, et al. Dysregulation of RUNX2/Activin-A Axis Upon miR-376c Downregulation Promotes Lymph Node Metastasis in Head and Neck Squamous Cell Carcinoma. Cancer Res (2016) 76(24):7140-50. doi: 10.1158/0008-5472.CAN-16-1188

14. de Carvalho AC, Scapulatempo-Neto C, Maia DC, Evangelista AF, Morini MA, Carvalho AL, et al. Accuracy of microRNAs as Markers for the Detection of Neck Lymph Node Metastases in Patients With Head and Neck Squamous Cell Carcinoma. BMC Med (2015) 13:108. doi: 10.1186/s12916-015-0350-3
RJJ, grant \# 2019HXBH079, \#2020SCU12049); The Science and Technology Department of Sichuan Province (YZ, grant \# 2020YFH0090, RJJ, grant\#2020YFS0111); The Health Department of Sichuan Province (JR, grant \# 20PJ030); China Postdoctoral Science Foundation (JR, grant \# 2020M673250); The Foundation of National Clinical Research Center for Geriatrics (SH, grant \# Z20201013); and National Natural Youth Science Foundation of China (JR, grant \# 82002868).

\section{SUPPLEMENTARY MATERIAL}

The Supplementary Material for this article can be found online at: https://www.frontiersin.org/articles/10.3389/fonc.2021.711171/ full\#supplementary-material

15. Hiyoshi Y, Kamohara H, Karashima R, Sato N, Imamura Y, Nagai Y, et al MicroRNA-21 Regulates the Proliferation and Invasion in Esophageal Squamous Cell Carcinoma. Clin Cancer Res an Off J Am Assoc Cancer Res (2009) 15(6):1915-22. doi: 10.1158/1078-0432.CCR-08-2545

16. Huang WC, Chan SH, Jang TH, Chang JW, Ko YC, Yen TC, et al. miRNA491-5p and GIT1 Serve as Modulators and Biomarkers for Oral Squamous Cell Carcinoma Invasion and Metastasis. Cancer Res (2014) 74(3):751-64. doi 10.1158/0008-5472.CAN-13-1297

17. Huang WC, Jang TH, Tung SL, Yen TC, Chan SH, Wang LH. A Novel miR365-3p/EHF/keratin 16 Axis Promotes Oral Squamous Cell Carcinoma Metastasis, Cancer Stemness and Drug Resistance via Enhancing Beta5Integrin/C-Met Signaling Pathway. J Exp Clin Cancer Res CR (2019) 38 (1):89. doi: 10.1186/s13046-019-1091-5

18. Kong KL, Kwong DL, Chan TH, Law SY, Chen L, Li Y, et al. MicroRNA-375 Inhibits Tumour Growth and Metastasis in Oesophageal Squamous Cell Carcinoma Through Repressing Insulin-Like Growth Factor 1 Receptor. Gut (2012) 61(1):33-42. doi: 10.1136/gutjnl-2011-300178

19. Liberati A, Altman DG, Tetzlaff J, Mulrow C, Gøtzsche PC, Ioannidis JPA, et al. The PRISMA Statement for Reporting Systematic Reviews and MetaAnalyses of Studies That Evaluate Health Care Interventions: Explanation and Elaboration. J Clin Epidemiol (2009) 62(10):e1-e34. doi: 10.1016/ j.jclinepi.2009.06.006

20. Whiting PF, Rutjes AW, Westwood ME, Mallett S, Deeks JJ, Reitsma JB, et al. QUADAS-2: A Revised Tool for the Quality Assessment of Diagnostic Accuracy Studies. Ann Intern Med (2011) 155(8):529-36. doi: 10.7326/ 0003-4819-155-8-201110180-00009

21. Tierney JF, Stewart LA, Ghersi D, Burdett S, Sydes MR. Practical Methods for Incorporating Summary Time-to-Event Data Into Meta-Analysis. Trials (2007) 8:16-. doi: 10.1186/1745-6215-8-16

22. Ahmad P, Sana J, Slavik M, Gurin D, Radova L, Gablo NA, et al. MicroRNA15b-5p Predicts Locoregional Relapse in Head and Neck Carcinoma Patients Treated With Intensity-Modulated Radiotherapy. Cancer Genomics Proteomics (2019) 16(2):139-46. doi: 10.21873/cgp.20119

23. Bonnin N, Armandy E, Carras J, Ferrandon S, Battiston-Montagne P, Aubry $\mathrm{M}$, et al. MiR-422a Promotes Loco-Regional Recurrence by Targeting NT5E/ CD73 in Head and Neck Squamous Cell Carcinoma. Oncotarget (2016) 7 (28):44023-38. doi: 10.18632/oncotarget.9829

24. Ganci F, Sacconi A, Bossel Ben-Moshe N, Manciocco V, Sperduti I, Strigari L, et al. Expression of TP53 Mutation-Associated microRNAs Predicts Clinical Outcome in Head and Neck Squamous Cell Carcinoma Patients. Ann Oncol Off J Eur Soc Med Oncol (2013) 24(12):3082-8. doi: 10.1093/annonc/mdt380

25. Ganci F, Sacconi A, Manciocco V, Covello R, Benevolo M, Rollo F, et al. Altered Peritumoral microRNA Expression Predicts Head and Neck Cancer Patients With a High Risk of Recurrence. Modern Pathol an Off J United States Can Acad Pathol Inc (2017) 30(10):1387-401. doi: 10.1038/ modpathol.2017.62 
26. Ganci F, Sacconi A, Manciocco V, Sperduti I, Battaglia P, Covello R, et al. MicroRNA Expression as Predictor of Local Recurrence Risk in Oral Squamous Cell Carcinoma. Head Neck (2016) 38 Suppl 1:E189-97. doi: 10.1002/hed.23969

27. Harris T, Jimenez L, Kawachi N, Fan J-B, Chen J, Belbin T, et al. Low-Level Expression of miR-375 Correlates With Poor Outcome and Metastasis While Altering the Invasive Properties of Head and Neck Squamous Cell Carcinomas. Am J Pathol (2012) 180(3):917-28. doi: 10.1016/j.ajpath.2011.12.004

28. Hudcova K, Raudenska M, Gumulec J, Binkova H, Horakova Z, Kostrica R, et al. Expression Profiles of miR-29c, miR-200b and miR-375 in Tumour and Tumour-Adjacent Tissues of Head and Neck Cancers. Tumour Biol J Int Soc Oncodevelopmental Biol Med (2016) 37(9):12627-33. doi: 10.1007/s13277016-5147-2

29. He T, Guo X, Li X, Liao C, Wang X, He K. Plasma-Derived Exosomal microRNA-130a Serves as a Noninvasive Biomarker for Diagnosis and Prognosis of Oral Squamous Cell Carcinoma. J Oncol (2021) 2021:5547911. doi: $10.1155 / 2021 / 5547911$

30. Rajthala S, Dongre H, Parajuli H, Min A, Nginamau ES, Kvalheim A, et al. Combined In Situ Hybridization and Immunohistochemistry on Archival Tissues Reveals Stromal microRNA-204 as Prognostic Biomarker for Oral Squamous Cell Carcinoma. Cancers (Basel) (2021) 13(6):1370. doi: 10.3390/ cancers 13061307

31. Song J, Zhang N, Cao L, Xiao D, Ye X, Luo E, et al. Down-Regulation of miR200c Associates With Poor Prognosis of Oral Squamous Cell Carcinoma. Int $J$ Clin Oncol (2020) 25(6):1072-8. doi: 10.1007/s10147-020-01649-2

32. Re M, Ceka A, Rubini C, Ferrante L, Zizzi A, Gioacchini FM, et al. MicroRNA$34 c-5 p$ is Related to Recurrence in Laryngeal Squamous Cell Carcinoma. Laryngoscope (2015) 125(9):E306-12. doi: 10.1002/lary.25475

33. Re M, Magliulo G, Gioacchini FM, Bajraktari A, Bertini A, Ceka A, et al. Expression Levels and Clinical Significance of miR-21-5p, miR-Let-7a, and miR-34c-5p in Laryngeal Squamous Cell Carcinoma. BioMed Res Int (2017) 2017:3921258. doi: 10.1155/2017/3921258

34. Ries J, Baran C, Wehrhan F, Weber M, Neukam FW, Krautheim-Zenk A, et al. Prognostic Significance of Altered miRNA Expression in Whole Blood of OSCC Patients. Oncol Rep (2017) 37(6):3467-74. doi: 10.3892/or.2017.5639

35. Liu N, Tang LL, Sun Y, Cui RX, Wang HY, Huang BJ, et al. MiR-29c Suppresses Invasion and Metastasis by Targeting TIAM1 in Nasopharyngeal Carcinoma. Cancer Lett (2013) 329(2):181-8. doi: 10.1016/j.canlet.2012. 10.032

36. Wang $\mathrm{H}$, Zhu $\mathrm{Y}$, Zhao $\mathrm{M}$, Wu C, Zhang P, Tang L, et al. miRNA-29c Suppresses Lung Cancer Cell Adhesion to Extracellular Matrix and Metastasis by Targeting Integrin $\beta 1$ and Matrix Metalloproteinase2 (MMP2). PloS One (2013) 8(8):e70192. doi: 10.1371/journal.pone.0070192

37. Wang L, Yu T, Li W, Li M, Zuo Q, Zou Q, et al. The miR-29c-KIAA1199 Axis Regulates Gastric Cancer Migration by Binding With WBP11 and PTP4A3. Oncogene (2019) 38(17):3134-50. doi: 10.1038/s41388-018-0642-0

38. Zhang JX, Mai SJ, Huang XX, Wang FW, Liao YJ, Lin MC, et al. MiR-29c Mediates Epithelial-to-Mesenchymal Transition in Human Colorectal Carcinoma Metastasis via PTP4A and GNA13 Regulation of $\beta$-Catenin Signaling. Ann Oncol (2014) 25(11):2196-204. doi: 10.1093/annonc/mdu439

39. Cheng YX, Zhang QF, Hong L, Pan F, Huang JL, Li BS, et al. MicroRNA-200b Suppresses Cell Invasion and Metastasis by Inhibiting the EpithelialMesenchymal Transition in Cervical Carcinoma. Mol Med Rep (2016) 13 (4):3155-60. doi: 10.3892/mmr.2016.4911

40. Humphries B, Wang Z, Li Y, Jhan JR, Jiang Y, Yang C. ARHGAP18 Downregulation by miR-200b Suppresses Metastasis of Triple-Negative Breast Cancer by Enhancing Activation of RhoA. Cancer Res (2017) 77 (15):4051-64. doi: 10.1158/0008-5472.CAN-16-3141

41. Li J, Yuan J, Yuan X, Zhao J, Zhang Z, Weng L, et al. MicroRNA-200b Inhibits the Growth and Metastasis of Glioma Cells via Targeting ZEB2. Int J Oncol (2016) 48(2):541-50. doi: 10.3892/ijo.2015.3267

42. Li Y, Guan B, Liu J, Zhang Z, He S, Zhan Y, et al. MicroRNA-200b is Downregulated and Suppresses Metastasis by Targeting LAMA4 in Renal Cell Carcinoma. EBioMedicine (2019) 44:439-51. doi: 10.1016/j.ebiom.2019. 05.041

43. Williams LV, Veliceasa D, Vinokour E, Volpert OV. miR-200b Inhibits Prostate Cancer EMT, Growth and Metastasis. PloS One (2013) 8(12): e83991. doi: 10.1371/journal.pone.0083991
44. Cao ZH, Cheng JL, Zhang Y, Bo CX, Li YL. MicroRNA-375 Inhibits Oral Squamous Cell Carcinoma Cell Migration and Invasion by Targeting Platelet -Derived Growth Factor-a. Mol Med Rep (2017) 15(2):922-8. doi: 10.3892/ mmr.2016.6057

45. Cui F, Wang S, Lao I, Zhou C, Kong H, Bayaxi N, et al. miR-375 Inhibits the Invasion and Metastasis of Colorectal Cancer via Targeting SP1 and Regulating EMT-Associated Genes. Oncol Rep (2016) 36(1):487-93. doi: 10.3892/or.2016.4834

46. Jimenez L, Sharma VP, Condeelis J, Harris T, Ow TJ, Prystowsky MB, et al. MicroRNA-375 Suppresses Extracellular Matrix Degradation and Invadopodial Activity in Head and Neck Squamous Cell Carcinoma. Arch Pathol Lab Med (2015) 139(11):1349-61. doi: 10.5858/arpa.2014-0471-OA

47. Wang F, Li Y, Zhou J, Xu J, Peng C, Ye F, et al. miR-375 is Down-Regulated in Squamous Cervical Cancer and Inhibits Cell Migration and Invasion via Targeting Transcription Factor SP1. Am J Pathol (2011) 179(5):2580-8. doi: 10.1016/j.ajpath.2011.07.037

48. Yang S, Yang R, Lin R, Si L. MicroRNA-375 Inhibits the Growth, Drug Sensitivity and Metastasis of Human Ovarian Cancer Cells by Targeting PAX2. J buon (2019) 24(6):2341-6.

49. Yi J, Jin L, Chen J, Feng B, He Z, Chen L, et al. MiR-375 Suppresses Invasion and Metastasis by Direct Targeting of SHOX2 in Esophageal Squamous Cell Carcinoma. Acta Biochim Biophys Sin (Shanghai) (2017) 49(2):159-69. doi: 10.1093/abbs/gmw131

50. Liu M, Xiusheng H, Xiao X, Wang Y. Overexpression of miR-422a Inhibits Cell Proliferation and Invasion, and Enhances Chemosensitivity in Osteosarcoma Cells. Oncol Rep (2016) 36(6):3371-8. doi: 10.3892/ or.2016.5182

51. Sun J, Chen Z, Xiong J, Wang Q, Tang F, Zhang X, et al. MicroRNA-422a Functions as a Tumor Suppressor in Glioma by Regulating the Wnt/ $\beta$-Catenin Signaling Pathway via RPN2. Oncol Rep (2020) 44(5):2108-20. doi: 10.3892/or.2020.7741

52. Wang $\mathrm{H}$, Tang $\mathrm{C}, \mathrm{Na} \mathrm{M}, \mathrm{Ma} \mathrm{W}$, Jiang $\mathrm{Z}$, Gu Y, et al. miR-422a Inhibits Glioma Proliferation and Invasion by Targeting IGF1 and IGF1R. Oncol Res (2017) 25 (2):187-94. doi: 10.3727/096504016X14732772150389

53. Zhang J, Yang Y, Yang T, Yuan S, Wang R, Pan Z, et al. Double-Negative Feedback Loop Between microRNA-422a and Forkhead Box (FOX)G1/Q1/E1 Regulates Hepatocellular Carcinoma Tumor Growth and Metastasis. Hepatology (2015) 61(2):561-73. doi: 10.1002/hep.27491

54. Chava S, Reynolds CP, Pathania AS, Gorantla S, Poluektova LY, Coulter DW, et al. miR-15a-5p, miR-15b-5p, and miR-16-5p Inhibit Tumor Progression by Directly Targeting MYCN in Neuroblastoma. Mol Oncol (2020) 14(1):180-96. doi: $10.1002 / 1878-0261.12588$

55. Chung TK, Lau TS, Cheung TH, Yim SF, Lo KW, Siu NS, et al. Dysregulation of microRNA-204 Mediates Migration and Invasion of Endometrial Cancer by Regulating FOXC1. Int J Cancer (2012) 130(5):1036-45. doi: 10.1002/ ijc. 26060

56. Fan X, Fang X, Liu G, Xiong Q, Li Z, Zhou W. MicroRNA-204 Inhibits the Proliferation and Metastasis of Breast Cancer Cells by Targeting PI3K/AKT Pathway. J buon (2019) 24(3):1054-9.

57. Hu WB, Wang L, Huang XR, Li F. MicroRNA-204 Targets SOX4 to Inhibit Metastasis of Lung Adenocarcinoma. Eur Rev Med Pharmacol Sci (2019) 23 (4):1553-62. doi: 10.26355/eurrev_201902_17114

58. Shi L, Zhang B, Sun X, Lu S, Liu Z, Liu Y, et al. MiR-204 Inhibits Human NSCLC Metastasis Through Suppression of NUAK1. Br J Cancer (2014) 111 (12):2316-27. doi: 10.1038/bjc.2014.580

59. Yang S, Chen B, Zhang B, Li C, Qiu Y, Yang H, et al. Mir-204-5p Promotes Apoptosis and Inhibits Migration of Gastric Cancer Cells by Targeting HER -2. Mol Med Rep (2020) 22(4):2645-54. doi: 10.3892/mmr.2020.11367

60. Yu Y, Wang Y, Xiao X, Cheng W, Hu L, Yao W, et al. MiR-204 Inhibits Hepatocellular Cancer Drug Resistance and Metastasis Through Targeting NUAK1. Biochem Cell Biol (2019) 97(5):563-70. doi: 10.1139/bcb-2018-0354

61. Zhang B, Cui H, Sun Y, Wang X, Jia Q, Li J, et al. Up-Regulation of miR-204 Inhibits Proliferation, Invasion and Apoptosis of Gallbladder Cancer Cells by Targeting Notch2. Aging (Albany NY) (2021) 13(2):2941-58. doi: 10.18632/ aging.202444

62. Cao W, Zhao Y, Wang L, Huang X. Circ0001429 Regulates Progression of Bladder Cancer Through Binding miR-205-5p and Promoting VEGFA Expression. Cancer biomark (2019) 25(1):101-13. doi: 10.3233/CBM-182380 
63. De Cola A, Lamolinara A, Lanuti P, Rossi C, Iezzi M, Marchisio M, et al. MiR-205$5 p$ Inhibition by Locked Nucleic Acids Impairs Metastatic Potential of Breast Cancer Cells. Cell Death Dis (2018) 9(8):821. doi: 10.1038/s41419-018-0854-9

64. Lang Y, Xu S, Ma J, Wu J, Jin S, Cao S, et al. MicroRNA-429 Induces Tumorigenesis of Human Non-Small Cell Lung Cancer Cells and Targets Multiple Tumor Suppressor Genes. Biochem Biophys Res Commun (2014) 450 (1):154-9. doi: 10.1016/j.bbrc.2014.05.084

65. Doberstein K, Bretz NP, Schirmer U, Fiegl H, Blaheta R, Breunig C, et al. miR21-3p is a Positive Regulator of L1CAM in Several Human Carcinomas. Cancer Lett (2014) 354(2):455-66. doi: 10.1016/j.canlet.2014.08.020

66. Tseng HH, Tseng YK, You JJ, Kang BH, Wang TH, Yang CM, et al. NextGeneration Sequencing for microRNA Profiling: MicroRNA-21-3p Promotes Oral Cancer Metastasis. Anticancer Res (2017) 37(3):1059-66. doi: 10.21873/ anticanres. 11417

67. Chang RM, Yang H, Fang F, Xu JF, Yang LY. MicroRNA-331-3p Promotes Proliferation and Metastasis of Hepatocellular Carcinoma by Targeting PH Domain and Leucine-Rich Repeat Protein Phosphatase. Hepatology (2014) 60 (4):1251-63. doi: 10.1002/hep.27221

68. Chen HH, Zong J, Wang SJ. LncRNA GAPLINC Promotes the Growth and Metastasis of Glioblastoma by Sponging miR-331-3p. Eur Rev Med Pharmacol Sci (2019) 23(1):262-70. doi: 10.26355/eurrev_201901_16772

69. Sarkar A, Rahaman A, Biswas I, Mukherjee G, Chatterjee S, Bhattacharjee S, et al. Tgfß Mediated LINC00273 Upregulation Sponges Mir200a-3p and Promotes Invasion and Metastasis by Activating ZEB1. J Cell Physiol (2020) 235(10):7159-72. doi: 10.1002/jcp.29614

70. Feng S, Zhu X, Fan B, Xie D, Li T, Zhang X. Mir-19a-3p Targets PMEPA1 and Induces Prostate Cancer Cell Proliferation, Migration and Invasion. Mol Med Rep (2016) 13(5):4030-8. doi: 10.3892/mmr.2016.5033

71. Jiang XM, Yu XN, Liu TT, Zhu HR, Shi X, Bilegsaikhan E, et al. microRNA19a-3p Promotes Tumor Metastasis and Chemoresistance Through the PTEN/Akt Pathway in Hepatocellular Carcinoma. BioMed Pharmacother (2018) 105:1147-54. doi: 10.1016/j.biopha.2018.06.097

72. Li Q, Li B, Li Q, Wei S, He Z, Huang X, et al. Exosomal miR-21-5p Derived From Gastric Cancer Promotes Peritoneal Metastasis via Mesothelial-toMesenchymal Transition. Cell Death Dis (2018) 9(9):854. doi: 10.1038/ s41419-018-0928-8

73. Zhang R, Xia T. Long Non-Coding RNA XIST Regulates PDCD4 Expression by Interacting With miR-21-5p and Inhibits Osteosarcoma Cell Growth and Metastasis. Int J Oncol (2017) 51(5):1460-70. doi: 10.3892/ijo.2017.4127

74. Zhu Y, Bo H, Chen Z, Li J, He D, Xiao M, et al. LINC00968 can Inhibit the Progression of Lung Adenocarcinoma Through the miR-21-5p/SMAD7 Signal Axis. Aging (Albany NY) (2020) 12(21):21904-22. doi: 10.18632/aging.104011

75. Liu H, Cheng Y, Xu Y, Xu H, Lin Z, Fan J, et al. The Inhibition of Tumor Protein P53 by microRNA-151a-3p Induced Cell Proliferation, Migration and Invasion in Nasopharyngeal Carcinoma. Biosci Rep (2019) 39(10): BSR20191357. doi: 10.1042/BSR20191357

76. Lu D, Tang L, Zhuang Y, Zhao P. miR-17-3P Regulates the Proliferation and Survival of Colon Cancer Cells by Targeting Par4. Mol Med Rep (2018) 17 (1):618-23. doi: 10.3892/mmr.2017.7863

77. Yang X, Du WW, Li H, Liu F, Khorshidi A, Rutnam ZJ, et al. Both Mature miR-17-5p and Passenger Strand miR-17-3p Target TIMP3 and Induce Prostate Tumor Growth and Invasion. Nucleic Acids Res (2013) 41 (21):9688-704. doi: 10.1093/nar/gkt680

78. Wang YY, Yan L, Yang S, Xu HN, Chen TT, Dong ZY, et al. Long Noncoding RNA AC073284.4 Suppresses Epithelial-Mesenchymal Transition by Sponging miR-18b-5p in Paclitaxel-Resistant Breast Cancer Cells. J Cell Physiol (2019) 234(12):23202-15. doi: 10.1002/jcp.28887
79. Yang Y, Xia S, Zhang L, Wang W, Chen L, Zhan W. MiR-324-5p/PTPRD/ CEBPD Axis Promotes Papillary Thyroid Carcinoma Progression via Microenvironment Alteration. Cancer Biol Ther (2020) 21(6):522-32. doi: 10.1080/15384047.2020.1736465

80. Liu ZM, Wu ZY, Li WH, Wang LQ, Wan JN, Zhong Y. MiR-96-5p Promotes the Proliferation, Invasion and Metastasis of Papillary Thyroid Carcinoma Through Down-Regulating CCDC67. Eur Rev Med Pharmacol Sci (2019) 23 (8):3421-30. doi: 10.26355/eurrev_201904_17706

81. Wei S, Zheng Y, Jiang Y, Li X, Geng J, Shen Y, et al. The circRNA circPTPRA Suppresses Epithelial-Mesenchymal Transitioning and Metastasis of NSCLC Cells by Sponging miR-96-5p. EBioMedicine (2019) 44:182-93. doi: 10.1016/ j.ebiom.2019.05.032

82. Zhang H, Chen R, Shao J. MicroRNA-96-5p Facilitates the Viability, Migration, and Invasion and Suppresses the Apoptosis of Cervical Cancer Cells Bynegatively Modulating Sfrp4. Technol Cancer Res Treat (2020) 19:1533033820934132. doi: 10.1177/1533033820934132

83. Chen J, Yan D, Wu W, Zhu J, Ye W, Shu Q. MicroRNA-130a Promotes the Metastasis and Epithelial-Mesenchymal Transition of Osteosarcoma by Targeting PTEN. Oncol Rep (2016) 35(6):3285-92. doi: 10.3892/or.2016.4719

84. Gregory PA, Bert AG, Paterson EL, Barry SC, Tsykin A, Farshid G, et al. The miR-200 Family and miR-205 Regulate Epithelial to Mesenchymal Transition by Targeting ZEB1 and SIP1. Nat Cell Biol (2008) 10(5):593-601. doi: 10.1038/ ncb1722

85. Chen H, Li Z, Zhang L, Zhang L, Zhang Y, Wang Y, et al. MicroRNA-200c Inhibits the Metastasis of Triple-Negative Breast Cancer by Targeting ZEB2, an Epithelial-Mesenchymal Transition Regulator. Ann Clin Lab Sci (2020) 50 (4):519-27.

86. Tan T, Xu XH, Lu XH, Wang XW. MiRNA-200a-3p Suppresses the Proliferation, Migration and Invasion of Non-Small Cell Lung Cancer Through Targeting IRS2. Eur Rev Med Pharmacol Sci (2020) 24(2):712-20. doi: 10.26355/eurrev_202001_20050

87. Chen J, Wang L, Matyunina LV, Hill CG, McDonald JF. Overexpression of miR-429 Induces Mesenchymal-to-Epithelial Transition (MET) in Metastatic Ovarian Cancer Cells. Gynecol Oncol (2011) 121(1):200-5. doi: 10.1016/ j.ygyno.2010.12.339

88. Hou X, Yang L, Jiang X, Liu Z, Li X, Xie S, et al. Role of microRNA-141-3p in the Progression and Metastasis of Hepatocellular Carcinoma Cell. Int J Biol Macromol (2019) 128:331-9. doi: 10.1016/j.ijbiomac.2019.01.144

Conflict of Interest: The authors declare that the research was conducted in the absence of any commercial or financial relationships that could be construed as a potential conflict of interest.

Publisher's Note: All claims expressed in this article are solely those of the authors and do not necessarily represent those of their affiliated organizations, or those of the publisher, the editors and the reviewers. Any product that may be evaluated in this article, or claim that may be made by its manufacturer, is not guaranteed or endorsed by the publisher.

Copyright (c) 2021 Qiu, Song, Rao, Liu, Cheng, Pang, Ren and Zhao. This is an openaccess article distributed under the terms of the Creative Commons Attribution License (CC BY). The use, distribution or reproduction in other forums is permitted, provided the original author(s) and the copyright owner(s) are credited and that the original publication in this journal is cited, in accordance with accepted academic practice. No use, distribution or reproduction is permitted which does not comply with these terms. 